\title{
New (re)purpose for an old drug: purinergic modulation may extinguish the COVID-19 thromboinflammatory firestorm
}

\author{
Yogendra Kanthi, ${ }^{1,2}$ Jason S. Knight, ${ }^{3}$ Yu Zuo, ${ }^{3}$ and David J. Pinsky ${ }^{1,4}$ \\ 1Division of Cardiovascular Medicine, Department of Internal Medicine, University of Michigan, Ann Arbor, Michigan, USA \\ ${ }^{2}$ Section of Vascular Thrombosis and Inflammation, National Heart, Lung, and Blood Institute, NIH, Bethesda, Maryland, \\ USA. ${ }^{3}$ Division of Rheumatology, Department of Internal Medicine and ${ }^{4}$ Department of Molecular and Integrative \\ Physiology, University of Michigan, Ann Arbor, Michigan, USA.
}

\section{Background}

Severe manifestations of coronavirus disease 2019 (COVID-19), caused by severe acute respiratory syndrome coronavirus 2 (SARS-CoV-2), such as acute respiratory distress syndrome (ARDS), are characterized by hyperinflammation, exuberant cytokine release, profound and progressive hypoxia, deranged coagulation, and multiorgan failure (1). COVID-19 also presents not infrequently with a devastating thrombotic diathesis, manifesting as arterial thrombosis, pulmonary embolism, deep vein thrombosis, and thrombotic microangiopathy. Not surprisingly, dysregulation of tonic vascular homeostatic functions of endothelial and hematopoietic lineage cells has been implicated in the pathophysiology of COVID-19. Reminiscent of neutrophil and macrophage hyperactivation syndromes, the COVID-19-associated systemic inflammatory response syndrome is likely driven by the activation and/or death of infected cells and collateral damage triggered by explosive production of cytokines and chemokines. Recruited leukocytes spew a fibrin/platelet-entrapping meshwork of DNA and associated histones, which in a vicious cycle further recruits leukocytes and triggers intravascular coagulation through endothelial, platelet, and leukocyte dysfunction (2). These concepts are supported by autopsy studies that have detected clusters of activated and degenerating myeloid cells in both intravascular and extravascular spaces (3) as well as an endotheliitis that is characterized by viral inclusion bodies and SARS-CoV-2 nucleoprotein in the walls of blood vessels $(4,5)$.

\section{Immunothrombosis in COVID-19}

Autopsy specimens from patients with overwhelming SARS-CoV-2 infection have demonstrated histopathologic findings of pulmonary microvascular thrombosis (3). When combined with the clinical observations of ventilation-perfusion mismatching and resultant profound hypoxemia, renal failure, pulmonary emboli, ischemic stroke, mesenteric ischemia, and digital necrosis, a common theme emerges - microvascular and macrovascular accretion of thrombus contributes to patient demise. Biomarker studies have revealed a marked imbalance of coagulation and fibrinolysis, which has emerged as a compelling marker of disease severity in COVID-19. Elevated levels of D-dimer, a fibrin(ogen) degradation product, are a hallmark of patients hospitalized with moderate and severe COVID-19, where they predict not only sever-

Conflict of interest: YK has served as a consultant to Surface Oncology and Acer Therapeutics. JSK has received research funding from Jazz Pharma for preclinical studies of defibrotide. YK and DJP have a pending patent on the use of biogases in cardiovascular disease (USPTO application US20180369278A1).

Copyright: () 2020, American Society for Clinical Investigation.

Reference information: /CI Insight. 2020;5(14):e140971. https://doi.org/10.1172/jici. insight.140971. ity, but also mortality (6). Furthermore, greater than $25 \%$ of patients with severe COVID- 19 will suffer a thrombotic complication characterized by accrual of intravascular fibrin, leukocytes, platelets, and erythrocytes (2). Thrombosis in critical illnesses such as ARDS occurs at the convergence of inflammation and coagulation. Endothelial cells release Weibel-Palade bodies containing vWF, along with a preformed membrane-based storage pool of cell adhesion proteins. Monocytes undergo pyroptosis, liberating the prototypical inflammatory cytokine IL-1 $\beta$, in addition to shedding microvesicles bearing procoagulant tissue factor $(5,7)$. Platelets activate neutrophils and self-aggregate in response to ADP and thrombin. Neutrophils release cytokines and extracellular chromatin traps (NETs) decorated with oxidant enzymes and microbicidal proteins. If not contained or degraded, NETs may function as damage-associated molecular patterns to amplify inflammation and thrombosis (8). Indeed, sera from patients with COVID-19 potently trigger healthy neutrophils to undergo NETosis, demonstrating a potential mechanism by which soluble factors in blood may remotely trigger immunothrombosis. Not surprisingly, molecular signatures of neutrophil hyperactivity correlate with clinical thrombosis and the requirement for mechanical ventilation in COVID-19 (9-11). Taken together, these findings provide a framework to understand and target 


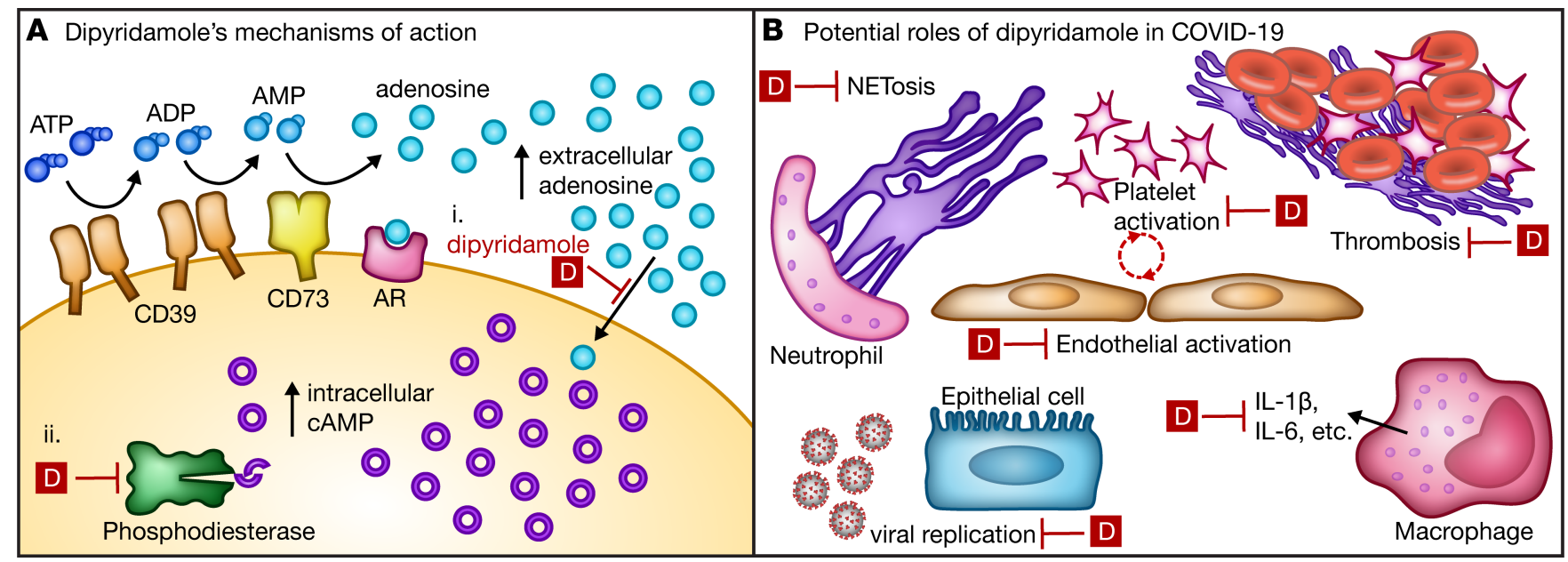

Figure 1. Dipyridamole's mechanisms of action and potential role in the treatment of COVID-19. (A) Dipyridamole potentiates homeostatic adenosine receptor signaling by preventing reuptake of ectonucleoside (i) and by stabilizing the intracellular cAMP pools via inhibition of cytoplasmic phosphodiesterase (PDE) activity (ii). (B) Left unchecked, purinergic signaling exacerbates both inflammation and thrombosis; therefore, dipyridamole has the potential to inhibit multiple processes that have been recently linked to COVID-19 severity. Illustrated by Rachel Davidowitz.

the thromboinflammation that drives severe disease and mortality in COVID-19. Based on the lynchpin role that purinergic nucleotides (ATP, ADP) play in platelet and leukocyte activation and the contribution of the nucleoside adenosine to endothelial and leukocyte quiescence, we hypothesize that harnessing purinergic signaling could amplify vascular homeostasis and potentially limit some of the devastating clinical sequelae driven at the nexus of inflammation and coagulation.

\section{Targeting purinergic signaling to prevent thromboinflammation}

Purinergic signaling represents a critical checkpoint in the self-amplifying thromboinflammatory loop of endothelial dysfunction and hyperactivation of neutrophils, monocytes, and platelets. Activated and injured cells release their stores of ATP and ADP, creating a purinergic cloud in the extracellular space, where these nucleotides function in an autocrine and paracrine fashion as "danger" signals for neighboring and downstream tissues. Activation of extracellular ATP receptors (such as P2X7) on monocytes and neutrophils engages the canonical NLRP3 inflammasome, resulting in exuberant IL-1 $\beta$ production $(12,13)$. Moreover, blockade of this pathway prevents accretion of venous and arterial thrombi in mice (14-16). Similarly, thienopyridine antagonists of the ADP P2Y12 receptor are used clinically to suppress platelet activation in patients with cardiovascular disease (17).

CD39 and CD73, the dominant vascular ectonucleotidases, sequentially phosphohydrolyze these nucleotides to generate adenosine, which sustains homeostasis and a quiescent, antiinflammatory microenvironment. Furthermore, efficient activation of the extracellular adenosine receptor $2 \mathrm{~A}\left(\mathrm{~A}_{2 \mathrm{~A}} \mathrm{R}\right)$ by the FDA-approved drug dipyridamole tempers both neutrophil ROS formation and NET release in a cAMPand PKA-dependent manner, while preventing intraluminal vascular thrombi in mice $(18,19)$. Dipyridamole also protects the endothelium from ROS generation and acquiring a tissue factor-rich procoagulant phenotype induced by TNF- $\alpha$ (20-22). Dipyridamole potentiates homeostatic adenosine receptor signaling through at least 2 mechanisms: (a) inhibition of ectonucleoside reuptake and (b) stabilization of intracellular cAMP via inhibition of cytoplasmic phosphodiesterase activity (Figure 1). Dipyridamole is an old and relatively safe drug, with FDA-approved indications for use in combination with aspirin to prevent recurrent strokes and in combination with vitamin $\mathrm{K}$ antagonists for thromboprophylaxis following mechanical heart valve replacement (23). Dipyridamole was once one of the fifty most prescribed drugs in the US (24), with a favorable safety profile and a bleeding risk similar to that of aspirin.

In addition to its antithrombotic properties, human and animal studies have demonstrated that dipyridamole both induces synthesis of antiviral type I IFNs and protects against harmful inflammatory responses in the context of endotoxemia $(25,26)$. Intriguingly, dipyridamole has recently been shown to inhibit SARS-CoV-2 replication in vitro, likely via direct interaction with the SARS-CoV-2 replication complex at concentrations below those achieved in plasma with FDA-approved dosing. In a small study in 
China, dipyridamole significantly suppressed D-dimer elevation and improved platelet counts in patients with COVID-19; however, larger studies are needed to evaluate clinical outcomes (27).

\section{COVID-19 therapy on (re)purpose: purinergic modulation in clinical trials}

There remains a paucity of drugs approved to treat the thromboinflammatory milieu of COVID-19. Current trials to address this problem either block specific inflammatory cytokines or broadly paralyze the coagulation system. For example, protocols using inhibitors of IL-6 signaling are ongoing in patients with severe disease, while a large observational study conducted in New York has suggested that therapeutic anticoagulation with heparin may reduce mortality in patients with severe COVID-19 (albeit with little effect on patients with mild or moderate disease) (28). To date, marked reductions in mortality or progression of COVID-19 to the severest complications such as ARDS have not been observed. The lack of noted effect may suggest that these treatments are incomplete, as each drug targets only one facet of a complex local and systemic thromboinflammatory milieu (29).

These observations provide a rationale for pursuing purinergic modulators, such as dipyridamole, as a means to amplify the body's natural homeostatic pathways and, in so doing, target the myriad cellular and molecular pathways that appear to be relevant to COVID-19 pathogenesis. In particular, prospective randomized clinical trials would be necessary to determine whether dipyridamole has a therapeutic benefit in the treatment of patients with COVID-19. Along with others, our group is developing such protocols to launch multiple clinical trials with dipyridamole at different stages of COVID-19 (NCT04391179), which will be made available on http://clinicaltrials.gov. If successful in clinical trials, purinergic modulation will be an accessible and scalable approach to break the relentless and lethal cycle of thromboinflammation in COVID-19.

\section{Author contributions}

YK, JSK, YZ, and DJP participated in the writing of the manuscript and gave approval before submission.

\section{Acknowledgments}

The work was supported by a COVID-19 Cardiovascular Impact Research Ignitor Grant from the Michigan Medicine Frankel Cardiovascular Center as well as by the A. Alfred Taubman Medical Research Institute. YK was supported by the NIH (K08HL131993, R01HL150392), a Falk Medical Research Trust Catalyst Award, the JOBST-American Venous Forum Award, and, in part, by the Intramural Research Program of the NIH and National Heart, Lung, and Blood Institute. JSK was supported by grants from the NIH (R01HL134846, R01HL150392), Lupus Research Alliance, and Burroughs Wellcome Fund. YZ was supported by career development grants from the Rheumatology Research Foundation and APS ACTION. DJP was supported by the NIH (R01HL150392). The authors thank all members of the "NETwork to Target Neutrophils in COVID-19" for their helpful advice and encouragement.

Address correspondence to: Yogendra Kanthi, National Heart, Lung, and Blood Institute, National Institutes of Health, 10 Center Drive, Room 5-5130, Bethesda, Maryland 20892, USA. Phone: 734.998.7955; Email: yogen.kanthi@nih.gov. Or to: Jason S. Knight, University of Michigan, 5560 MSRB2, 1150 West Medical Center Drive, Ann Arbor, Michigan 48109, USA. Phone: 734.936.3257; Email: jsknight@umich. edu. Or to: David J. Pinsky, University of Michigan, 2141 Cardiovascular Center, 1500 East Medical Center Drive, Ann Arbor, Michigan 48109, USA. Phone: 734.936.3500; Email: dpinsky@umich.edu.

1. Huang C, et al. Clinical features of patients infected with 2019 novel coronavirus in Wuhan, China. Lancet. 2020;395(10223):497-506.

2. Klok FA, et al. Incidence of thrombotic complications in critically ill ICU patients with COVID-19. Thromb Res. 2020;191:145-147.

3. Fox SE, Akmatbekov A, Harbert JL, Li G, Quincy Brown J, Vander Heide RS. Pulmonary and cardiac pathology in African American patients with COVID-19: an autopsy series from New Orleans. Lancet Respir Med. 2020;8(7):681-686

4. Varga Z, et al. Endothelial cell infection and endotheliitis in COVID-19. Lancet. 2020;395(10234):1417-1418.

5. Merad M, Martin JC. Pathological inflammation in patients with COVID-19: a key role for monocytes and macrophages. Nat Rev Immunol. 2020;20(6):355-362.

6. Zhang L, et al. D-dimer levels on admission to predict in-hospital mortality in patients with Covid-19. J Thromb Haemost. 2020;18(6):1324-1329.

7. Wu C, et al. Inflammasome activation triggers blood clotting and host death through oyroptosis. Immunity. 2019;50(6):1401-1411.e4. 
8. von Brühl ML, et al. Monocytes, neutrophils, and platelets cooperate to initiate and propagate venous thrombosis in mice in vivo. J Exp Med. 2012;209(4):819-835.

9. Shi H, et al. Neutrophil calprotectin identifies severe pulmonary disease in COVID-19. medRxiv. https://doi.org/10.1101/2020 .05.06.20093070. Published May 17, 2020. Accessed June 25, 2020.

10. Zuo Y, et al. Neutrophil extracellular traps in COVID-19. JCI Insight. 2020;5(11):138999.

11. Zuo Y, et al. Neutrophil extracellular traps and thrombosis in COVID-19. medRxiv. https://doi.org/10.1101/2020.04.30.20086736. Published May 29, 2020. Accessed June 25, 2020.

12. Carta S, et al. Cell stress increases ATP release in NLRP3 inflammasome-mediated autoinflammatory diseases, resulting in cytokine imbalance. Proc Natl Acad Sci USA. 2015;112(9):2835-2840.

13. Karmakar M, Katsnelson MA, Dubyak GR, Pearlman E. Neutrophil P2X7 receptors mediate NLRP3 inflammasome-dependent IL-1 $\beta$ secretion in response to ATP. Nat Commun. 2016;7:10555.

14. Yadav V, et al. Ectonucleotidase tri(di)phosphohydrolase-1 (ENTPD-1) disrupts inflammasome/interleukin 1 $\beta$-driven venous thrombosis. J Clin Invest. 2019;129(7):2872-2877.

15. Furlan-Freguia C, Marchese P, Gruber A, Ruggeri ZM, Ruf W. P2X7 receptor signaling contributes to tissue factor-dependent thrombosis in mice. J Clin Invest. 2011;121(7):2932-2944.

16. Anyanwu AC, et al. Tuning the thromboinflammatory response to venous flow interruption by the ectonucleotidase CD39. Arterioscler Thromb Vasc Biol. 2019;39(4):e118-e129.

17. Yusuf S, et al. Effects of clopidogrel in addition to aspirin in patients with acute coronary syndromes without ST-segment elevation. N Engl J Med. 2001;345(7):494-502.

18. Ali RA, et al. Adenosine receptor agonism protects against NETosis and thrombosis in antiphospholipid syndrome. Nat Commun 2019;10(1):1916.

19. Cronstein BN, Sitkovsky M. Adenosine and adenosine receptors in the pathogenesis and treatment of rheumatic diseases Nat Rev Rheumatol. 2017;13(1):41-51.

20. Chakrabarti S, Vitseva O, Iyu D, Varghese S, Freedman JE. The effect of dipyridamole on vascular cell-derived reactive oxygen species. J Pharmacol Exp Ther. 2005;315(2):494-500.

21. Deguchi H, Takeya H, Urano H, Gabazza EC, Zhou H, Suzuki K. Adenosine regulates tissue factor expression on endothelial cells. Thromb Res. 1998;91(2):57-64.

22. Guo S, Stins M, Ning M, Lo EH. Amelioration of inflammation and cytotoxicity by dipyridamole in brain endothelial cells. Cerebrovasc Dis. 2010;30(3):290-296.

23. Persantine (dipyridamole) [package insert]. Ridgefield, CT: Boehringer Ingelheim Pharmaceuticals, Inc. December 2019.

24. FitzGerald GA. Dipyridamole. N Engl J Med. 1987;316(20):1247-1257.

25. Galabov AS, Mastikova M. Dipyridamole induces interferon in man. Biomed Pharmacother. 1984;38(8):412-413.

26. Ramakers BP, et al. Dipyridamole augments the antiinflammatory response during human endotoxemia. Crit Care. 2011;15(6):R289.

27. Liu X, et al. Potential therapeutic effects of dipyridamole in the severely ill patients with COVID-19 [published ahead of print April 20, 2020]. Acta Pharm Sin B. https://doi.org/10.1016/j.apsb.2020.04.008.

28. Paranjpe I, et al. Association of Treatment Dose Anticoagulation with In-Hospital Survival Among Hospitalized Patients with COVID-19 [published ahead of print May 5, 2020]. J Am Coll Cardiol. https://doi.org/10.1016/j.jacc.2020.05.001.

29. Colling ME, Kanthi Y. COVID-19-associated coagulopathy: An exploration of mechanisms [published ahead of print June 19 2020]. Vasc Med. https://doi.org/10.1177/1358863X20932640. 